\title{
Clinical Outcomes of Surgical Management of Primary Brachial Plexus Tumors
}

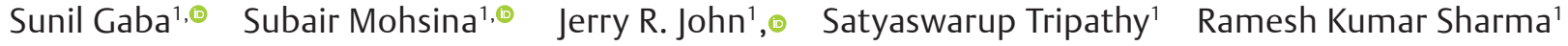 \\ ${ }^{1}$ Department of Plastic Surgery, Postgraduate Institute of Medical \\ Education and Research (PGIMER), Chandigarh, India

\begin{abstract}
Address for correspondence Satyaswarup Tripathy, MCh, Department of Plastic Surgery, Postgraduate Institute of Medical Education and Research (PGIMER), Chandigarh 160012, India (e-mail: dr.sstripathy@gmail.com).
\end{abstract}

Indian J Plast Surg 2021;54:124-129.

\begin{abstract}
Introduction This study evaluates the clinical presentation, tumor characteristics, and clinical outcomes of surgically treated benign and malignant brachial plexus tumors (BPTs).

Methods A prospective study of patients with BPTs from June 2015 to August 2020 was conducted. All patients underwent surgical resection with microneurolysis and intraoperative electrical stimulation to preserve the functioning nerve fascicles.

Results Fourteen patients with 15 BPTs underwent surgical resection. Mean age was $37.8 \pm 12.3$ years; with male to female ratio $4: 10$. The clinical presentations were swelling (100\%), pain (84.6\%), and paresthesia (76.9\%). The lesions involved roots $(5 / 15)$, trunk (5/15), division (1/15), and cords (4/15). Thirteen patients had benign pathology (8 schwannomas, 3 neurofibromas, 2 lipomas) and two had malignant neurofibrosarcoma. Gross total resection was achieved in all cases except a dumbbell tumor.

Keywords

- schwannoma

- neurofibroma

- microneurolysis

- paresthesia

- dumbbell tumor The mean follow-up period was $24 \pm 5$ months. Postoperatively, all patients reported improvement in pain and paresthesia with no new sensory deficit. All patients had developed initial motor weakness (Grades 2-4); however, full power (Grade 5) was recovered by 3 to 5 months.

Conclusion Total resection can be achieved by appropriate microneural dissection and electrophysiologic monitoring and is potentially curative with preserving function.
\end{abstract}

\section{Introduction}

Brachial plexus tumors (BPTs) are rare and constitute $5 \%$ of all the upper-limb tumors. ${ }^{1,2}$ These include benign or malignant tumors arising from neural or surrounding nonneural tissues. ${ }^{3}$ Preoperative evaluation and planning depend on origin, characteristics, extension, and pathology of tumor. The tumor often causes displacement in the already complex neurovascular anatomy. Procedures such as tumor resection or even biopsy are prone to risk of losing limb functions thus making it a clinical and surgical challenge. Successful management of these lesions requires a thorough understanding of anatomy and familiarity with surgical variations that may be encountered.

published online June 28, 2021
DOI https://doi.org/

$10.1055 / \mathrm{s}-0041-1731252$

ISSN 0970-0358
The surgical management of BPTs has undergone a sea change from en-masse excision of tumor and nerve to enucleation of encapsulated tumors. ${ }^{4-6}$ The first description of tumor excision in 1886 resulted in significant morbidity. ${ }^{7}$ Refinements in surgical techniques aim at preservation of limb functions. The improved functional outcomes have been achieved with the help of better preoperative imaging, higher magnification, and intraoperative neural monitoring techniques. ${ }^{4}$ There are few recent reports demonstrating the surgical approaches and variations expected. ${ }^{4,8-12}$ However, most of these are either isolated case reports or series owing to the heterogeneity in clinicopathological presentations and is often limited by a retrospective design. This inevitably leads

(C) 2021. Association of Plastic Surgeons of India.

This is an open access article published by Thieme under the terms of the Creative Commons Attribution-NonDerivative-NonCommercial-License, permitting copying and reproduction so long as the original work is given appropriate credit. Contents may not be used for commercial purposes, or adapted, remixed, transformed or built upon. (https://creativecommons.org/licenses/by-nc-nd/4.0/).

Thieme Medical and Scientific Publishers Pvt. Ltd. A-12, 2nd Floor, Sector 2, Noida-201301 UP, India 
to frequent misdiagnosis and delay in management. ${ }^{11-13}$ With a thorough understanding of varied presentations and surgical challenges involved, the potential for preservation of limb functions can be achieved. Hence, this study was performed to describe the clinical presentation, tumor characteristics, and clinical outcomes of surgically treated primary BPTs.

\section{Methods}

This was a prospective descriptive analysis of patients with BPTs performed from June 2015 to August 2020. Institute Research Committee approval and informed consent were obtained from all patients. Fourteen patients who underwent surgical management for BPTs were included.

BPT was diagnosed based on clinical presentation, imaging, and tissue diagnosis. Patients presenting with swelling over neck, shoulder or axilla, with or without pain or tingling in upper-limb were suspected to have BPT. Detailed history regarding the progression of symptoms, size of swelling, relevant family history, presence of neurofibromatosis (NF1) and history of attempted excision were noted. Clinical examination of location, size, mobility, and tenderness of swelling and detailed neurological assessment was performed. All patients underwent magnetic resonance imaging (MRI) to define the tumor's location and the extent and its relation to the neural elements. In cases of dumbbell tumors involving roots and intravertebral foramina, computerized-tomography imaging was obtained. Tissue-diagnosis was performed with fine needle aspiration cytology (FNAC) for primary cases and histopathological review of previous biopsy slides if available.

\section{Surgical Technique}

All patients underwent surgical exploration under magnification. An anterior supraclavicular and infraclavicular approach was used for upper lesions (roots/trunks) and lower lesions (divisions/ cords/terminal branches), respectively. The axillary incision extended onto medial arm was used to access the distal end of axillary lesions. The authors were able to gain wide access with this combined approach and the need for clavicle resection was avoided in all the cases. The tumor was identified in relation to plexus and mobilized from adjacent structures. The nerves proximal and distal to the lesion were identified and protected using silicon vascular slings.

The thickened epineurium over the lesion was opened, entering extracapsular plane carefully until mass was separated from adjacent nerve fascicles. Fascicles entering the substance of the tumor were isolated at proximal and distal poles of mass. ${ }^{4,8}$ Intraoperative electrical stimulation $(0.5-2 \mathrm{~mA} ; 2 \mathrm{~Hz})$ was used to differentiate functioning and nonfunctioning fascicles within the tumor. Microneurolysis was performed under $10 \mathrm{X}$ magnification to preserve the functioning nerve fascicles, which were displaced, splayed out, or thinned due to the mass effect of expanded tumor. Smaller tumors could be shelled out as a single mass easily with careful microneural dissection. However, very large tumors often needed debulking over nonneural areas to help in the ease of dissection. Once adequate debulking was achieved, the plane of cleavage between tumor and functioning nerve fascicles was identified and tumor was excised completely. Malignant tumors were excised with $1 \mathrm{~cm}$ margin of surrounding tissue to ensure complete oncological clearance. The nerves were included in the excision if required to achieve free margins and secondary functional reconstruction procedures such as distal nerve/tendon transfers were planned.

\section{Postoperative Protocol}

Patients were immobilized with crepe bandage dressings for upto 3 weeks postoperatively. The patient was started on passive range of motion exercises for 1 week and followed up by active range of motion exercises. In case of motor deficit postoperatively, passive physiotherapy and galvanic current electrical stimulation were applied till M2 power was recovered and was then followed by active range of motion exercises.

\section{Statistical Analysis}

All the continuous variables were expressed as means and categorical variables were expressed as proportions. Descriptive analysis was performed using SPSS software V.21.

\section{Results}

\section{Clinical and Demographic Characteristics}

Fourteen patients underwent surgical management of 15 BPTs. Four male and ten female patients were included with a mean age of $37.8 \pm 12.3$ years (range: $16-55$ years). Majority of patients $(66.6 \%)$ were index cases except 4 patients who had history of attempted excision at outside centers. None of the patients had history of neurofibromatosis. The symptom duration ranged from 3 months to 1 year. The most common clinical presentations were swelling (100\%), pain (86.6\%), and paresthesia (76.9\%). Sensory and motor deficits were present in 5 and 4 patients, respectively. Tinel's sign could be elicited at location of tumor in 11/15 cases. The summary of the various clinicopathological features of the patients is shown in - Table 1.

\section{Tumor Characteristics}

The tumors were varied in their location, extent, and pathological characteristics. MRI demonstrated ovoid mass along the nerves with homogenous enhancement in T1 and hyperintense with postcontrast enhancement on T2-weighted images ( - Fig. 1). Malignant lesions were characterized by large size, irregular peripheral enhancement, and intratumoral cystic appearance ( - Fig. 1). The lesions involved various parts of brachial plexus such as roots (5/15), trunk (5/15), division (1/15), and cords (4/15) (-Table1) One patient had bilateral tumor (Schwannoma) involving all the roots on right and divisions on left side (-Fig. 2). One patient had dumbell-shaped tumor having intraspinal and extraspinal extension onto C5 root (-Fig. 3). Thirteen patients had benign pathology: 8 schwannomas, 3 neurofibromas, and 2 lipomas. Two patients had malignant neurofibrosarcoma.

\section{Intraoperative Features}

All tumors were excised as a single mass with preservation of fascicles. Gross total resection (GTR) was achieved 
Table 1 Summary of the various clinicopathological features of the patients in the series

\begin{tabular}{|c|c|c|c|c|c|c|c|c|}
\hline No. & $\begin{array}{l}\text { Age/ } \\
\text { gender }\end{array}$ & Clinical symptoms & $\begin{array}{l}\text { Tinel's } \\
\text { sign }\end{array}$ & $\begin{array}{l}\text { Preoperative } \\
\text { neural deficit }\end{array}$ & Location & Excision & $\begin{array}{l}\text { Postoperative } \\
\text { histopathology }\end{array}$ & $\begin{array}{l}\text { Postoperative neural } \\
\text { deficit }\end{array}$ \\
\hline 1 & $45 / F$ & $\begin{array}{l}\text { Swelling, pain, } \\
\text { paresthesia }\end{array}$ & + & $\begin{array}{l}\text { Sensory, } \\
\text { motor }\end{array}$ & C5 root & GTR & Schwannoma & \\
\hline 2 & $49 / F$ & $\begin{array}{l}\text { Swelling, pain, } \\
\text { paresthesia }\end{array}$ & + & & C5, 6 root & GTR & Schwannoma & \\
\hline 3 & $45 / F$ & $\begin{array}{l}\text { Swelling, pain, } \\
\text { paresthesia }\end{array}$ & + & & C5 root & GTR & Schwannoma & \\
\hline 4 & $16 / \mathrm{F}$ & Swelling, paresthesia & - & & $\begin{array}{l}\text { Upper } \\
\text { trunk }\end{array}$ & GTR & Schwannoma & \\
\hline 5 & $55 / \mathrm{M}$ & Swelling, pain & + & Motor & $\begin{array}{l}\text { Upper } \\
\text { trunk }\end{array}$ & GTR & Neurofibroma & \\
\hline 6 & $45 / \mathrm{M}$ & $\begin{array}{l}\text { Swelling, pain, } \\
\text { paresthesia }\end{array}$ & + & & $\begin{array}{l}\text { Middle } \\
\text { trunk }\end{array}$ & GTR & Lipoma & \\
\hline 7 & $46 / \mathrm{M}$ & $\begin{array}{l}\text { Swelling, pain, } \\
\text { paresthesia }\end{array}$ & + & Sensory & $\begin{array}{l}\text { Middle } \\
\text { trunk }\end{array}$ & GTR & Schwannoma & \\
\hline 8 & $24 / F$ & $\begin{array}{l}\text { Swelling, pain, } \\
\text { paresthesia }\end{array}$ & & & Lower trunk & GTR & Neurofibroma & \\
\hline \multirow[t]{2}{*}{9} & $30 / F^{a}$ & $\begin{array}{l}\text { Swelling, pain, } \\
\text { paresthesia }\end{array}$ & + & Sensory & C5-T1 roots & GTR & Schwannoma & Horner's syndrome \\
\hline & & & & & All division & GTR & Schwannoma & \\
\hline 10 & $22 / \mathrm{M}$ & Swelling, paresthesia & + & & Medial cord & GTR & Neurofibrosarcoma & \\
\hline 11 & $26 / F$ & Swelling, pain & & Sensory & Medial cord & GTR & Lipoma & \\
\hline 12 & $45 / F$ & $\begin{array}{l}\text { Swelling, pain, } \\
\text { paresthesia }\end{array}$ & + & & Medial cord & GTR & Schwannoma & \\
\hline 13 & $48 / \mathrm{F}$ & Swelling, pain & + & $\begin{array}{l}\text { Sensory, } \\
\text { motor }\end{array}$ & $\begin{array}{l}\text { C5.6 root } \\
\text { dumbbell } \\
\text { tumor }\end{array}$ & $\mathrm{STR}^{\mathrm{b}}$ & Neurofibroma & \\
\hline 14 & $44 / F$ & Swelling, pain & & Motor & Medial cord & GTR & Neurofibrosarcoma & $\begin{array}{l}\text { Index finger and thumb } \\
\text { flexion weakness(Grade 3) }\end{array}$ \\
\hline
\end{tabular}

Abbreviations: GTR, gross total resection, STR, subtotal resection.

apatient with bilateral tumor.

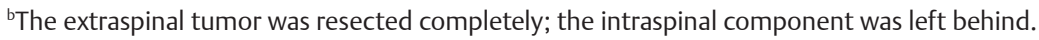

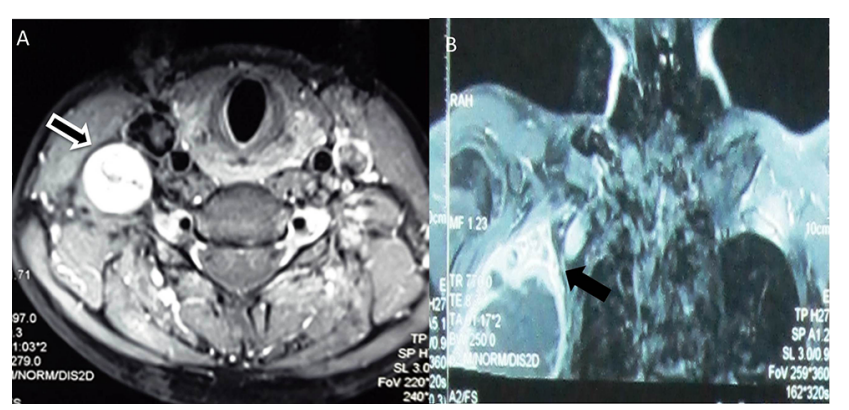

Fig. 1 Characteristic magnetic resonance imaging (MRI) findings of brachial plexus tumors: (A) Benign brachial plexus tumor-ovoid lesion along the axis of the nerve, hyperintense with postcontrast enhancement showing the target sign in T2-weighted images (black arrow). (B) Malignant tumor-MRI shows characteristic irregular peripheral enhancement and intratumoral cystic appearance (black arrow).

in $14 / 15$ cases. Schwannomas appeared as firm well-defined masses without much deformation of nerve and could be easily dissected out preserving neural tissues (-Fig. 2). Neurofibroma resulted in firm masses with splaying and enlargement of neural tissue making the dissection tedious (-Fig. 4). Electrical stimulation to identify and preserve functional fascicles was performed meticulously. In the patient with dumbbell tumor, subtotal resection (STR) was performed leaving behind intraspinal part of tumor ( - Fig. 3 ). In one case with a large tumor with cystic degeneration due to central tumor necrosis, debulking of the benign tumor by aspirating the contents was performed that facilitated retrieval of the deflated tumor, thus preserving function. In malignant peripheral nerve sheath tumor (MPNST) patients, GTR with $1 \mathrm{~cm}$ margin was achieved in one case with preservation of all neural fascicles, whereas another patient required partial excision of median nerve fascicles and adventitia of axillary vessels ( $\boldsymbol{- \text { Fig. }}$ 4). A line diagram representing the differences in dissection and excision between the various types of tumors is shown in $\boldsymbol{- F i g} \mathbf{5}$.

\section{Postoperative Recovery Characteristics}

The mean follow-up period was $24 \pm 5$ months. Postoperatively, all patients reported improvement in pain and paresthesia. None of the patients developed new sensory/motor deficit. All patients had developed initial motor weakness (Grades 2-4) in the muscles supplied by the involved/adjacent nerves; however, full power (Grade 5 ) was recovered in all those muscles by 3 to 5 months. For example, patient with 


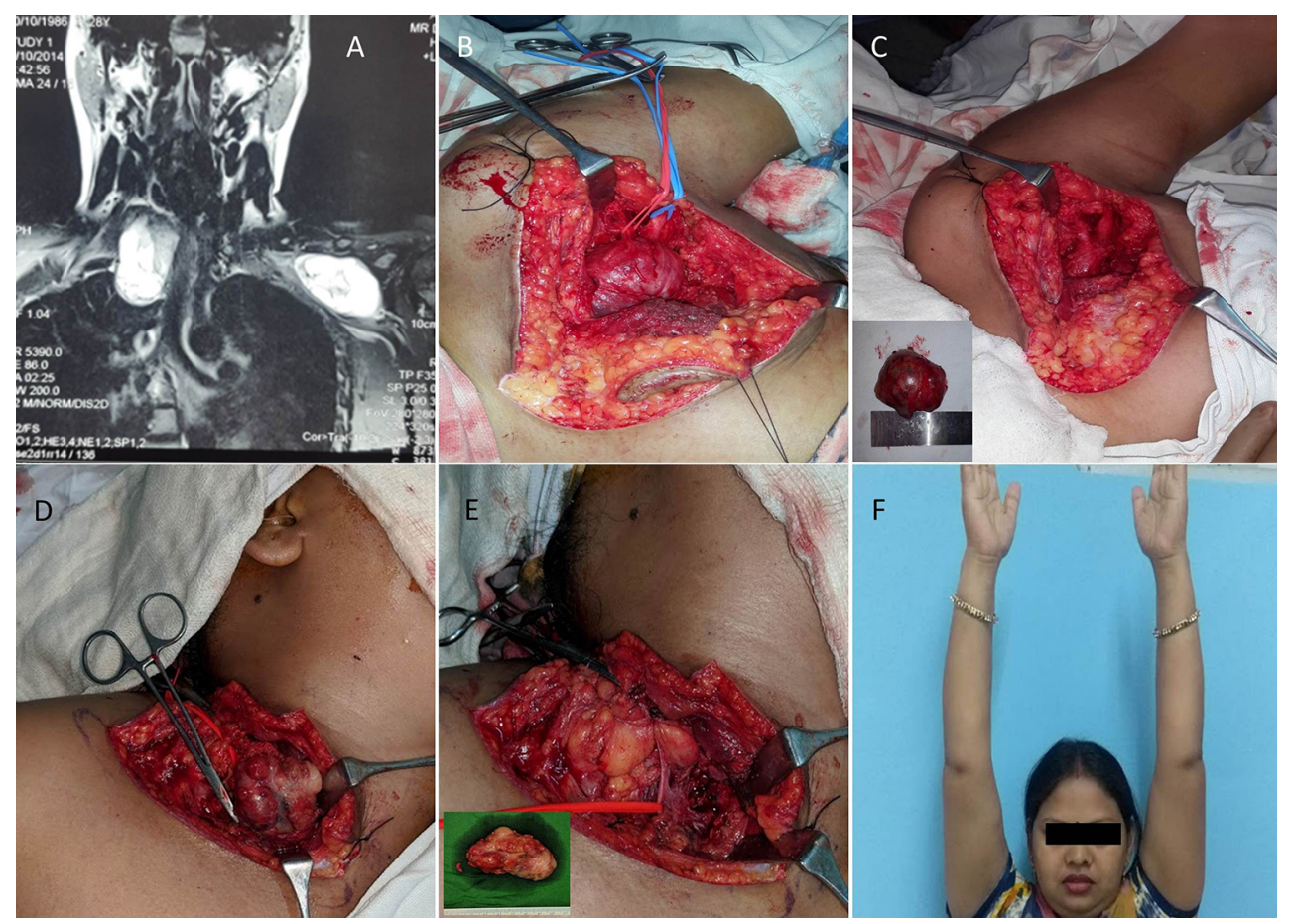

Fig. 2 A 30-year-old female with bilateral brachial plexus schwannoma. The patient was more symptomatic on the left side that was addressed first. (A) Coronal section showing bilateral tumors involving the C5-T1 roots on right side and all divisions on the left side. (B) Intraoperative picture demonstrating tumor of division in infraclavicular region. (C) Tumor excised in toto (inset) with preservation of neural tissue. The right side was addressed in the second stage. (D) Intraoperative photograph showing the tumor that was partly cystic. (E) Tumor excised in toto (inset) with preservation of neural tissue. (F) complete recovery of motor functions with persistent Horner's syndrome on the right side.

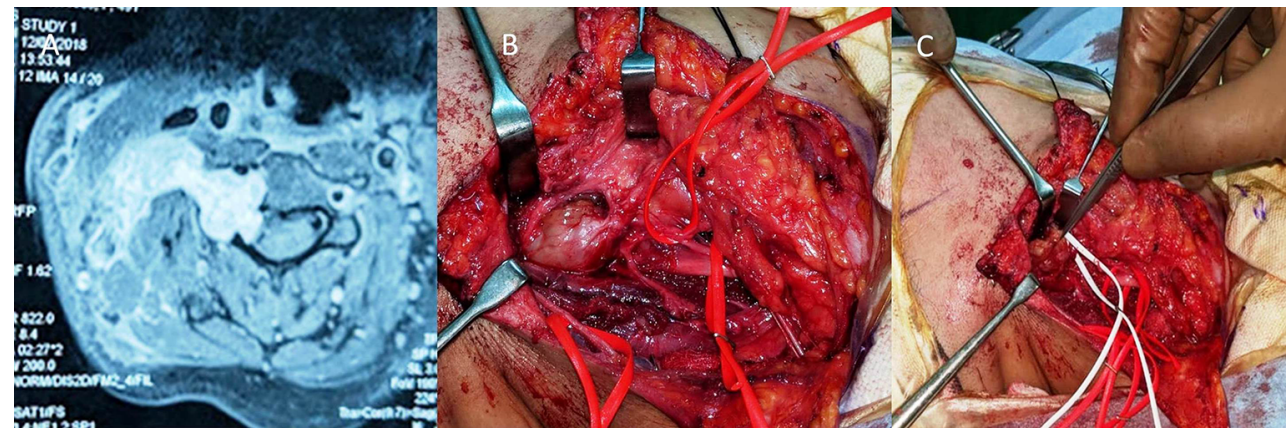

Fig. 3 Patient with dumbbell tumor of C5 root: (A) Magnetic resonance imaging showing the dumbbell-shaped tumor with intraspinal and extraspinal component on to C5 root. (B) Intraoperative picture showing the extraspinal component. (C) Intraoperative photograph demonstrating post subtotal resection of the tumor. Note the residual tumor in close relation and continuity onto intraspinal part.

tumor arising from C5 root had postoperative weakness of shoulder abduction; however, it improved to full power by 3 to 5 months. The patient with bilateral tumor had residual Horner's syndrome postoperatively on one side. Patient with dumbbell tumor who underwent STR reported improvement in sensory and motor deficits as well as myelopathy symptoms. One patient with malignancy succumbed to disease due to systemic metastasis at 9 months follow-up. Second patient had persistent motor deficit in the form of weakness (Grade 3) of index finger and thumb flexion. None of the patients developed recurrence.

\section{Discussion}

The primary BPTs constitute an unfamiliar and surgically challenging territory. With a better understanding of anatomy and surgical variations, the goal of curative resection with preservation of limb function is feasible. ${ }^{8,11,14,15}$ The first report of excision of brachial plexus schwannoma resulted in significant deltoid paralysis. ${ }^{7}$ Further studies reported significant postoperative neurological deficit or STRs. ${ }^{16-19}$ There has been constant modifications in the surgical technique with the increasing use of intraoperative neural monitoring and magnification aimed at improving outcomes and minimizing morbidity. There are few large series demonstrating the variations and surgical challenges. ${ }^{8-13,15}$ Huang et al reported a series of 20 cases with good outcomes. ${ }^{8}$ Recent reports from China and India also demonstrated the importance of appropriate surgical management to protect limb functions. ${ }^{11,13}$ However, majority of these reports were limited by sample size and retrospective design. 


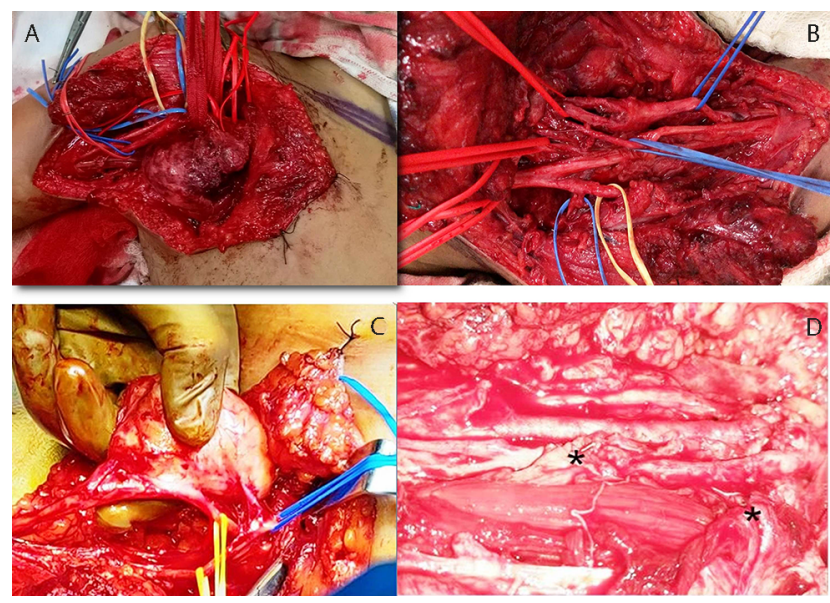

Fig. 4 Upper row: A 24-year-female with neurofibroma of medial cord (A), Preserved neural tissue after total excision of tumor by microneurolysis (B). Lower row: A 44-year-female with neurofibrosarcoma in relation to the origin of median nerve $(\mathbf{C})$. Excision with 1 $\mathrm{cm}$ margin requiring partial excision of the median nerve ( ${ }^{*}$ resected nerve ends) (D)

Current understanding of the approach to BPTs remains incomplete. This is reflected in a large number of misdiagnosed cases despite characteristic clinical signs. Jia et al reported $>50 \%$ patients who were wrongly diagnosed and treated as cervical spondylosis thus leading to delay in presentation to a specialist. ${ }^{13}$ Desai reported $12 \%$ cases misdiagnosed as either nonneural tumor or lymph nodes and underwent incomplete excision and abandonment of procedure. ${ }^{11}$ Similarly, in the present study $28 \%$ cases were diagnosed following previous attempted excision and biopsy for mistaken neck swellings. Although a rare diagnosis, this underscores the potential need to create awareness and have a high index of suspicion.

The mean age at diagnosis was 45 to 48 years in the previous reports; however, a relatively lower age was reported in the present study as well as in previous reports from India. ${ }^{8-11,15}$ Among benign BPTs, neurofibroma was the most common followed by schwannoma in reports from West. ${ }^{8-10,15,20,21}$ The recent series of 143 cases from China reported $83 \%$ schwannoma and $8 \%$ neurofibromas. ${ }^{13}$ Desai also reported 60\% schwannomas and 40\% neurofibromas. ${ }^{11}$ Similar results were noted in the present study with $50 \%$ cases being schwannoma. This geographical variation in epidemiology and tumor type is surprising and may be attributed to genetic or environmental factors.

A palpable mass was the most common clinical presentation (70-90\%) followed by pain (50-60\%) in majority of the reports and in the present study as well. ${ }^{8-15}$ The motor and sensory loss was a relatively rare presentation. Among patients with neurological deficits, diagnosis was predominantly neurofibroma than schwannoma. ${ }^{8-11}$ However, Huang et al reported pain and sensory loss as the major symptom (60\%) as opposed to palpable mass (30\%). ${ }^{15}$ Tinel's sign can differentiate these tumors from other neck swellings. In the present study, a positive Tinel's sign was elicited in $73 \%$ cases. Similar reports were shown by Desai and Jia et al. ${ }^{11,13}$

MRI has now been widely accepted as imaging of choice. Although it is difficult to differentiate pathological types of benign tumors, differentiation of benign and malignant lesions is possible on MRI.,22 In the present study, the authors have utilized a combination of MRI and FNAC. The core-needle biopsy was avoided as the risk of worsening of neurological symptoms with the needle trauma is larger. ${ }^{23}$ Although there is no definite role preoperatively, the importance of intraoperative electrodiagnostic studies to help in identification of functioning fascicles has been stressed in literature. , $^{811,13,15}$

Conservative management with serial MRI monitoring can be used for small tumors without any neurologic or cosmetic issues. However, surgical resection is potentially curative. Relative contraindications to surgery include pregnancy and a poor surgical candidate. Palpable tumors, intractable pain, neurological deficits, and suspicion of malignancy warrant early surgical management. Schwannomas arise from
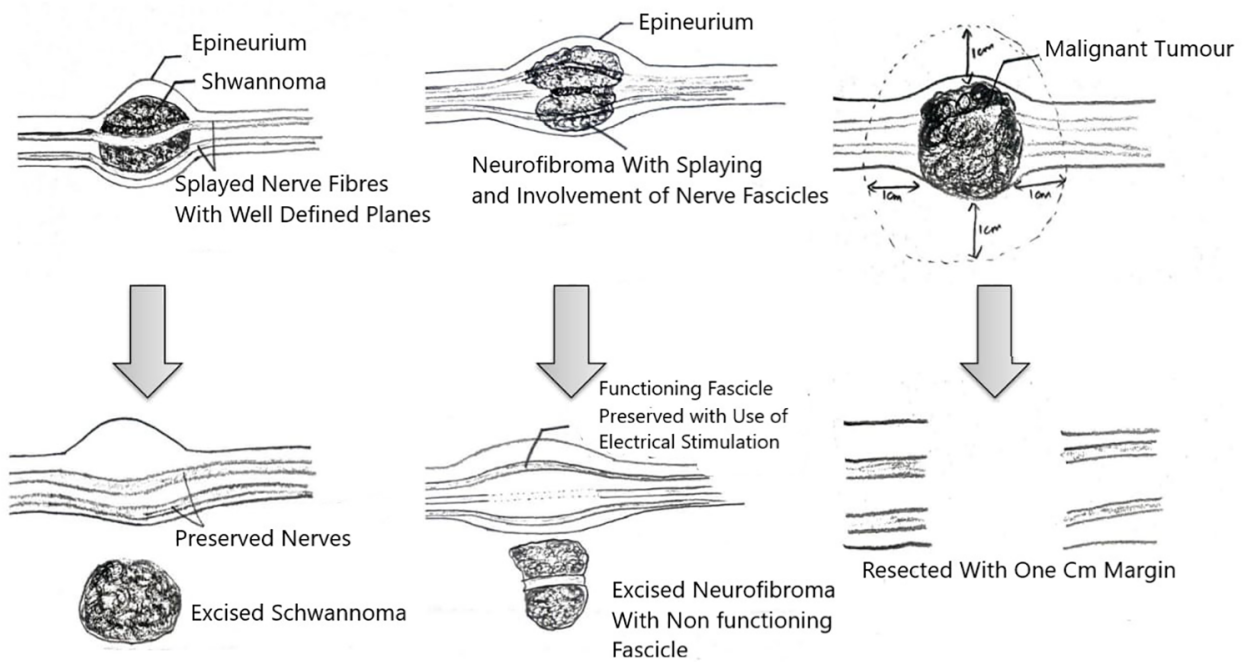

Fig. 5 Line diagram depicting the difference in dissection between benign (schwannoma and neurofibroma) and malignant brachial plexus tumors. 
Schwann cells and is eccentrically located within the nerve. They are soft to firm in consistency and may contain cystic or hemorrhagic areas. Schwannomas characteristically spare neural fascicles except few which enter and exit the tumors. They have a definite capsule formed of perineurium and fibrous epineurium and hence, intrafascicular dissection and isolation of tumor are relatively easy., ${ }^{8,10,12}$ Neurofibromas are firm, arise from perineurial cells, and lack a definite capsule. ${ }^{8,11,15}$ They involve larger number of neural fascicles intimately and have a higher risk of loss of neural functions. The older literature distinguished these two entities based on the need for nerve-resection and grafting in neurofibroma. ${ }^{4-6}$ However, with improvised techniques and intraoperative electrical stimulation, it is possible to excise both with careful preservation of functional neurons.

Huang et al reported improvement in pain without any sensory or neural deficit in $82 \%$ neurofibroma and $89 \%$ schwannomas. ${ }^{15}$ Ganju et al reported preservation of functions with total resection in $76 \%$ neurofibromas; however, in most of NF1 associated neurofibromas, nerve function could be preserved only at the cost of STR. ${ }^{8}$ Desai demonstrated total excision was possible in $65 \%$ tumors, with STR in $35 \%$ cases that were neurofibromas. ${ }^{11}$ In the present study, GTR could be achieved in 14/15 cases. Although combined supine and prone approach for complete excision of dumbbell tumors has been described, in the present series, STR was performed for dumbbell tumor. The patients did not require further excision of intraspinal part as the patient improved symptomatically. Previous reports demonstrated staged excision of dumbbell tumors and reported that addressing the extraspinal component was important to prevent recurrence. ${ }^{11}$ Jia et al reported complete recovery of neural functions in $93 \%$ patients with total or STR; however, 6\% reported persistent neurological deficits. ${ }^{13}$ Desai demonstrated $95 \%$ improvement in pain and paresthesia within weeks; however, 5/115 patients had persistent sensorimotor deficits. ${ }^{11}$ Similar results were reported with resolution of pain and paresthesia in early postoperative period in all patients in this study. However, all the patients had initial motor weakness owing to neurapraxia due to the intrafascicular dissection and handling of the nerve. Siqueira and Go et al also reported similar transient paralysis following total resection of benign tumors. ${ }^{10,12}$ In one patient with MPNST who required partial excision of median nerve fascicles, primary nerve reconstruction was not performed as adjuvant radiotherapy was planned. Although nerve resection and reconstruction is feasible, it was deferred keeping in mind the fact that reoperating on a postradiotherapy bed is technically difficult and is likely to have suboptimal outcomes. The study is not without limitations. The follow-up was performed by clinical examination for signs of recurrence and a postoperative MRI could not be performed. However, no recurrence of benign tumors has been reported in up to 5 years follow-up..$^{11,13}$

\section{Conclusion}

Total resection of BPTs can be achieved by appropriate microsurgical dissection and electrophysiologic monitoring and is potentially curative with excellent results.

\section{Conflict of Interests}

None.

\section{References}

1 Stack HG. Tumors of the hand. BMJ 1960;1(5177):919-922

2 Mackinnon SE, Dellon AL, Nerve repair and nerve grafts. In: Mackinnon SE, Dellon AL, eds. Surgery of the Peripheral Nerve. New York, NY; Thieme Medical Publishers; 1988 89-129

3 Lusk MD, Kline DG, Garcia CA. Tumors of the brachial plexus. Neurosurgery 1987;21(4):439-453

4 Huang JH, Samadani U, Zager EL. Brachial plexus region tumors: a review of their history, classification, surgicalmanagement, and outcomes. Neurosurg Q 2003;13(3):151-161

5 Nelaton U. Correspondence to the Societie de Chirurgie. Gazette de l'Hopital. 1864;37:307-308

6 Dubuisson AS, Kline DG. Brachial plexus injury: a survey of 100 consecutive cases from a single service. Neurosurgery 2002;51(3):673-682, discussion 682-683

7 Abe K, Takeuchi A, Yamamoto N, et al. Symptomatic small schwannoma is a risk factor for surgical complications and correlates with difficulty of enucleation. Springerplus 2015;4:751

8 Ganju A, Roosen N, Kline DG, Tiel RL. Outcomes in a consecutive series of 111 surgically treated plexal tumors: a review of the experience at the Louisiana State University Health Sciences Center. J Neurosurg 2001;95(1):51-60

9 Kim YW, Ahn SK, Song JH. A case of brachial plexus schwannoma. J Korean Neurosurg Soc 2006;39(5):396-399

10 Siqueira MG, Martins RS, Teixeira MJ. Management of brachial plexus region tumours and tumour-like conditions: relevant diagnostic and surgical features in a consecutive series of eighteen patients. Acta Neurochir (Wien) 2009;151(9):1089-1098

11 Desai KI. Primary benign brachial plexus tumors: an experience of 115 operated cases. Neurosurgery 2012;70(1):220-233, discussion 233

12 Go MH, Kim SH, Cho KH; GoMH. Brachial plexus tumors in a consecutive series of twenty one patients. J Korean Neurosurg Soc 2012;52(2):138-143

13 Jia X, Yang J, Chen L, Yu C, Kondo T. Primary brachial plexus tumors: clinical experiences of 143 cases. Clin Neurol Neurosurg 2016;148:91-95

14 Lee HJ, Kim JH, Rhee SH, Gong HS, Baek GH. Is surgery for brachial plexus schwannomas safe and effective? Clin Orthop Relat Res 2014;472(6):1893-1898

15 Huang JH, Zaghloul K, Zager EL. Surgical management of brachial plexus region tumors. Surg Neurol 2004;61(4):372-378

16 Godwin JT. Encapsulated neurilemoma (schwannoma) of the brachial plexus; report of eleven cases. Cancer 1952;5(4):708-720

17 Kline DG, Judice DJ. Operative management of selected brachial plexus lesions. J Neurosurg 1983;58(5):631-649

18 Fisher RG, Tate HB. Isolated neurilemomas of the brachial plexus. J Neurosurg 1970;32(4):463-467

19 Dart LH Jr, MacCarty CS, Love JG, Dockerty MB. Neoplasms of the brachial plexus. Minn Med 1970;53(9):959-964

20 Donner TR, Voorhies RM, Kline DG. Neural sheath tumors of major nerves. J Neurosurg 1994;81(3):362-373

21 Kehoe NJ, Reid RP, Semple JC. Solitary benign peripheral-nerve tumours. Review of 32 years' experience. J Bone Joint Surg Br 1995;77(3):497-500

22 Wasa J, Nishida Y, Tsukushi S, et al. MRI features in the differentiation of malignant peripheral nerve sheath tumors and neurofibromas. AJR Am J Roentgenol 2010;194(6):1568-1574

23 Pfadenhauer K, Grieser T, Scheglmann K. Percutaneous or open surgical biopsy in a case of symptomatic neoplastic brachial plexus infiltration? Ultrasound Int Open 2016;2(2):E71-E72 